\title{
On Polynomials and Their Polar Derivative
}

\author{
Abdullah Mir
}

(Communicated by Nihal YILMAZ ÖZGÜR)

\begin{abstract}
Let $P(z)$ be a polynomial of degree $n$ and for any complex number $\alpha$, let $D_{\alpha} P(z)=n P(z)+(\alpha-z) P^{\prime}(z)$ denote the polar derivative of $P(z)$ with respect to $\alpha$. Here, we consider the class of polynomials $P(z)=a_{n} z^{n}+\sum_{\nu=\mu}^{n} a_{n-\nu} z^{n-\nu}, 1 \leq \mu \leq n$, having all zeros in $|z| \leq k, k \leq 1$ and thereby establish several interesting results regarding the integral mean estimates for the polar derivative of $P(z)$. Our results not only generalize and refine some known polynomial inequalities, but also a variety of interesting results can be deduced from these by a fairly uniform procedure.
\end{abstract}

Keywords: Polar derivative, polynomials, zeros, $L^{\gamma}$-inequalities in the complex domain.

AMS Subject Classification (2010): Primary: 30A10 ; Secondary: 30C10; 30C15.

\section{Introduction and Statement of results.}

Let $P_{n}$ be the class of polynomials $P(z)=\sum_{\nu=0}^{n} a_{\nu} z^{\nu}$ of degree $n$. For $P \in P_{n}$, define

$$
\|P\|_{\gamma}:=\left\{\frac{1}{2 \pi} \int_{0}^{2 \pi}\left|P\left(e^{i \theta}\right)\right|^{\gamma}\right\}^{\frac{1}{\gamma}}, \gamma>0,\|P\|_{\infty}:=\max _{|z|=1}|P(z)|, \quad m:=\min _{|z|=k}|P(z)| \text { and } m_{1}:=\min _{|z|=1}|P(z)| .
$$

For fixed $\mu, 1 \leq \mu \leq n$, let $P_{n, \mu}$, denote the class of polynomials $P(z)=a_{n} z^{n}+\sum_{v=\mu}^{n} a_{n-v} z^{n-v}, 1 \leq \mu \leq n$, of degree $n$ having all zeros in $|z| \leq k, k \leq 1$.

If $P \in P_{n}$, then we have

$$
\left\|P^{\prime}\right\|_{\infty} \leq n\|P\|_{\infty}
$$

Equality holds in (1.1) if and only if $P(z)$ has all its zeros at the origin.

The above inequality (1.1) is better known as S. Bernestein's inequality (for example see [7]), although it first appeared in a paper of M. Riesz [[16], p.357].

For the class of polynomials $P \in P_{n}$ having all zeros in $|z| \leq 1$, Turán [17] proved that

$$
\left\|P^{\prime}\right\|_{\infty} \geq \frac{n}{2}\|P\|_{\infty}
$$

Inequality (1.2) was refined by Aziz and Dawood [2] and they proved under the same hypothesis that

$$
\left\|P^{\prime}\right\|_{\infty} \geq \frac{n}{2}\left\{\|P\|_{\infty}+m_{1}\right\}
$$

Received : 04-September-2015, Accepted : 15-June-2016 
Both the inequalities (1.2) and (1.3) are best possible and become equality for polynomials $P(z)=\alpha z^{n}+\beta$ where $|\alpha|=|\beta|$. As an extension of (1.2), it was shown by Malik [15], that if $P \in P_{n, 1}$, then

$$
\left\|P^{\prime}\right\|_{\infty} \geq \frac{n}{1+k}\|P\|_{\infty}
$$

where as the corresponding extension of (1.3) and a refinement of (1.4) was given by Govil [12] who under the same hypothesis proved that

$$
\left\|P^{\prime}\right\|_{\infty} \geq \frac{n}{1+k}\left\{\|P\|_{\infty}+\frac{m}{k^{n-1}}\right\} .
$$

In the literature, there already exist some refinements and generalizations of all the above inequalities, for example see Aziz and Shah [6], Dewan, Mir and Yadav [10], Govil, Rahman and Schemeisser [13], Dewan, Singh and Lal [8], etc.

Aziz and Shah [6] (see also Dewan, Mir and Yadav [10]) generalized inequality (1.5) and proved that, if $P \in P_{n, \mu}$, then

$$
\left\|P^{\prime}\right\|_{\infty} \geq \frac{n}{1+k^{\mu}}\left\{\|P\|_{\infty}+\frac{m}{k^{n-\mu}}\right\} .
$$

For $\mu=1$, inequality (1.6) reduces to inequality (1.5).

Let $D_{\alpha} P(z)$ denotes the polar derivative of the polynomial $P(z)$ of degree $n$ with respect to the point $\alpha \in \mathbb{C}$. Then

$$
D_{\alpha} P(z)=n P(z)+(\alpha-z) P^{\prime}(z) .
$$

The polynomial $D_{\alpha} P(z)$ is of degree at most $n-1$ and it generalizes the ordinary derivative in the sense that

$$
\lim _{\alpha \rightarrow \infty}\left\{\frac{D_{\alpha} P(z)}{\alpha}\right\}=P^{\prime}(z) .
$$

Aziz and Rather [5] extended (1.4) to the polar derivative of a polynomial and proved that if $P \in P_{n, 1}$, then for every complex number $\alpha$ with $|\alpha| \geq k$,

$$
\left\|D_{\alpha} P\right\|_{\infty} \geq n\left(\frac{|\alpha|-k}{1+k}\right)\|P\|_{\infty} .
$$

Aziz and Rather [4] refined inequality (1.7) and proved that if $P \in P_{n, \mu}$, then for every complex number $\alpha$ with $|\alpha| \geq s_{\mu}$,

$$
\left\|D_{\alpha} P\right\|_{\infty} \geq n\left(\frac{|\alpha|-s_{\mu}}{1+s_{\mu}}\right)\|P\|_{\infty}
$$

where

$$
s_{\mu}=\frac{n\left|a_{n}\right| k^{2 \mu}+\mu\left|a_{n-\mu}\right| k^{\mu-1}}{n\left|a_{n}\right| k^{\mu-1}+\mu\left|a_{n-\mu}\right|} .
$$

As a generalization of (1.6), Dewan, Singh and Lal [8] proved that if $P \in P_{n, \mu}$, then for every complex number $\alpha$ with $|\alpha| \geq k^{\mu}$,

$$
\left\|D_{\alpha} P\right\|_{\infty} \geq n\left(\frac{|\alpha|-k^{\mu}}{1+k^{\mu}}\right)\|P\|_{\infty}+\frac{n m(|\alpha|+1)}{k^{n-\mu}\left(1+k^{\mu}\right)} .
$$

Recently, Dewan et.al.[9] refined the above inequality (1.10) and proved for the same class of polynomials $P \in P_{n, \mu}$, that if $\alpha$ is any complex number with $|\alpha| \geq k^{\mu}$, then

$$
\left\|D_{\alpha} P\right\|_{\infty} \geq n\left(\frac{|\alpha|-A_{\mu}}{1+k^{\mu}}\right)\|P\|_{\infty}+\frac{m n}{k^{n}}\left(\frac{|\alpha| k^{\mu}+A_{\mu}}{1+k^{\mu}}\right),
$$

where

$$
A_{\mu}=\frac{n\left(\left|a_{n}\right|-\frac{m}{k^{n}}\right) k^{2 \mu}+\mu\left|a_{n-\mu}\right| k^{\mu-1}}{n\left(\left|a_{n}\right|-\frac{m}{k^{n}}\right) k^{\mu-1}+\mu\left|a_{n-\mu}\right|} .
$$

In the proof of the inequality (1.11) given by Dewan, Singh and Mir [9], the authors while using Laguerre's theorem claim to have deduced on page 814 that if $P(z)-\frac{m \lambda z^{n}}{k^{n}}$ has all its zeros in $|z|<k, k \leq 1$, then for $|\alpha| \geq k^{\mu}, 1 \leq \mu \leq n$, the polynomial $D_{\alpha}\left[P(z)-\frac{m \lambda z^{n}}{k^{n}}\right]$ also has all its zeros in $|z|<k, k \leq 1$, which is true when $|\alpha| \geq k$ and not for $|\alpha| \geq k^{\mu}, 1 \leq \mu \leq n$, in general. It is worth to mention that the result still follows without using Laguerre's theorem, for example see [1]. 


\section{Main Results}

In this paper, we shall prove some integral inequalities for the polar derivative of a polynomial having zeros in $|z| \leq k, k \leq 1$ and thereby obtain generalizations of many known results. We first prove the following interesting generalization of inequality (1.8).

Theorem 1.1. If $P \in P_{n, \mu}$, then for every complex number $\alpha$ with $|\alpha| \geq s_{\mu}$ and for each $\gamma>0, p>1, q>1$ with $p^{-1}+q^{-1}=1$, we have

$$
n\left(|\alpha|-s_{\mu}\right)\|P\|_{\gamma} \leq\left\|1+s_{\mu} z\right\|_{p \gamma}\left\|D_{\alpha} P\right\|_{q \gamma},
$$

where $s_{\mu}$ is defined by (1.9).

It is well known that $\|P\|_{\gamma}$ increases with $\gamma$ unless $P$ is a constant, and that $\lim _{\gamma \rightarrow \infty}\|P\|_{\gamma}=\max _{0 \leq \theta<2 \pi}\left|P\left(e^{i \theta}\right)\right|=\|$ $P \|_{\infty}$. If we let $\gamma \rightarrow \infty, p \rightarrow \infty$ (so that $q \rightarrow 1$ ) in (1.13), we get inequality (1.8).

Dividing both sides of (1.13) by $|\alpha|$ and let $|\alpha| \rightarrow \infty$, we get a result recently proved by Aziz and Rather ([3], Theorem 3). The following corollary immediately follows by letting $q \rightarrow \infty$ (so that $p \rightarrow 1$ ) in Theorem 1.1

Corollary 1.1. If $P \in P_{n, \mu}$, then for every complex number $\alpha$ with $|\alpha| \geq s_{\mu}$ and for each $\gamma>0$, we have

$$
n\left(|\alpha|-s_{\mu}\right)\|P\|_{\gamma} \leq\left\|1+s_{\mu} z\right\|_{\gamma}\left\|D_{\alpha} P\right\|_{\infty},
$$

where $s_{\mu}$ is defined by (1.9).

Dividing both sides of (1.14) by $|\alpha|$ and let $|\alpha| \rightarrow \infty$, we get a result of Aziz and Rather ([3], Corollary 5). If we take $\mu=1$ in Corollary 1.1, we get the following result.

Corollary 1.2. If $P \in P_{n, 1}$, then for every complex number $\alpha$ with $|\alpha| \geq s_{1}$ and for each $\gamma>0$,

$$
n\left(|\alpha|-s_{1}\right)\|P\|_{\gamma} \leq\left\|1+s_{1} z\right\|_{\gamma}\left\|D_{\alpha} P\right\|_{\infty}
$$

where

$$
s_{1}=\frac{n\left|a_{n}\right| k^{2}+\left|a_{n-1}\right|}{n\left|a_{n}\right|+\left|a_{n-1}\right|} .
$$

Remark 1.1. For $k=1$, Corollary 1.2 reduces to a result of Dewan, Singh, Mir and Bhat [11].

Next, we shall prove the following general result which provides a refinement of inequality (1.11) and hence of (1.10) as well. The interesting thing we shall see here is that the refinement of inequality (1.11) provided by Theorem 1.2 is independent of Laguerre's theorem.

Theorem 1.2. If $P \in P_{n, \mu}$, then for every complex numbers $\alpha, \beta$ with $|\alpha| \geq A_{\mu},|\beta|<1$ and for each $\gamma>0, p>1, q>1$ with $p^{-1}+q^{-1}=1$, we have

$$
n\left(|\alpha|-A_{\mu}\right)\left\|P-\frac{m \beta z^{n}}{k^{n}}\right\|_{\gamma} \leq\left\|1+A_{\mu} z\right\|_{p \gamma}\left\|D_{\alpha} P-\frac{\alpha \beta m n z^{n-1}}{k^{n}}\right\|_{q \gamma},
$$

where $A_{\mu}$ is defined by (1.12).

Remark 1.2. If we let $\gamma \rightarrow \infty$ and $p \rightarrow \infty$ (so that $q \rightarrow 1$ ) in (1.16), we get for $|\alpha| \geq A_{\mu}$ and $|\beta|<1$,

$$
\left\|D_{\alpha} P-\frac{\alpha \beta m n z^{n-1}}{k^{n}}\right\|_{\infty} \geq n\left(\frac{|\alpha|-A_{\mu}}{1+A_{\mu}}\right)\left\|P-\frac{m n \beta z^{n}}{k^{n}}\right\|_{\infty} .
$$

Let $z_{0}$ be a point on $|z|=1$ such that

$$
\left|D_{\alpha} P\left(z_{0}\right)-\frac{m n \alpha \beta z_{0}^{n-1}}{k^{n}}\right|=\max _{|z|=1}\left|D_{\alpha} P(z)-\frac{m n \alpha \beta z^{n-1}}{k^{n}}\right|,
$$


then from (1.17), we get for $|\alpha| \geq A_{\mu}$ and $|\beta|<1$,

$$
\left|D_{\alpha} P\left(z_{0}\right)-\frac{m n \alpha \beta z_{0}^{n-1}}{k^{n}}\right| \geq n\left(\frac{|\alpha|-A_{\mu}}{1+A_{\mu}}\right) \max _{|z|=1}\left|P(z)-\frac{m \beta z^{n}}{k^{n}}\right| .
$$

If in (1.18), we choose the argument of $\beta$ such that

$$
\left|D_{\alpha} P\left(z_{0}\right)-\frac{m n \alpha \beta z_{0}^{n-1}}{k^{n}}\right|=\left|D_{\alpha} P\left(z_{0}\right)\right|-\frac{m n|\alpha||\beta|\left|z_{0}\right|^{n-1}}{k^{n}},
$$

which is possible by Lemma 2.5 (stated in section 2), we get for $|\alpha| \geq A_{\mu}$ and $|z|=1$,

$$
\begin{gathered}
\left|D_{\alpha} P\left(z_{0}\right)\right|-\frac{m n|\alpha||\beta|\left|z_{0}\right|^{n-1}}{k^{n}} \geq n\left(\frac{|\alpha|-A_{\mu}}{1+A_{\mu}}\right)\left|P(z)-\frac{m \beta z^{n}}{k^{n}}\right| \\
\geq n\left(\frac{|\alpha|-A_{\mu}}{1+A_{\mu}}\right)\left\{|P(z)|-\frac{m|\beta|}{k^{n}}\right\} .
\end{gathered}
$$

Since $z_{0}$ lies on $|z|=1$, above inequality reduces to

$$
\left|D_{\alpha} P\left(z_{0}\right)\right| \geq n\left(\frac{|\alpha|-A_{\mu}}{1+A_{\mu}}\right)|P(z)|-n\left(\frac{|\alpha|-A_{\mu}}{1+A_{\mu}}\right) \frac{m|\beta|}{k^{n}}+\frac{m n|\alpha||\beta|}{k^{n}} \text { for }|z|=1 .
$$

Now, if in (1.20), we make $|\beta| \rightarrow 1$, we get for $|\alpha| \geq A_{\mu}$,

$$
\left\|D_{\alpha} P\right\|_{\infty} \geq n\left(\frac{|\alpha|-A_{\mu}}{1+A_{\mu}}\right)\|P\|_{\infty}+\frac{m n(|\alpha|+1) A_{\mu}}{k^{n}\left(1+A_{\mu}\right)} .
$$

Remark 1.3. Since by Lemma 2.3 (stated in Section 2) $A_{\mu} \leq k^{\mu}$, it follows that the inequality (1.21) holds for $|\alpha| \geq k^{\mu}$ as well.

It is easy to see that the inequality (1.21) provides a refinement of inequality (1.11) and hence of (1.10) as well. For this it needs to show that

$$
\begin{aligned}
& n\left(\frac{|\alpha|-A_{\mu}}{1+k^{\mu}}\right)\|P\|_{\infty}+\frac{m n}{k^{n}}\left(\frac{|\alpha| k^{\mu}+A_{\mu}}{1+k^{\mu}}\right) \\
& \leq n\left(\frac{|\alpha|-A_{\mu}}{1+A_{\mu}}\right)\|P\|_{\infty}+\frac{m n(|\alpha|+1) A_{\mu}}{k^{n}\left(1+A_{\mu}\right)}
\end{aligned}
$$

or

$$
\left(|\alpha|-A_{\mu}\right)\left(A_{\mu}-k^{\mu}\right)\|P\|_{\infty} \leq \frac{m}{k^{n}}\left\{A_{\mu}\left(|\alpha|-A_{\mu}\right)-k^{\mu}\left(|\alpha|-A_{\mu}\right)\right\} .
$$

Since $|\alpha| \geq A_{\mu}$ and $A_{\mu} \leq k^{\mu}$, above inequality is equivalent to

$$
\frac{m}{k^{n}} \leq\|P\|_{\infty}
$$

which is always true by Lemma 2.6 (stated in section 2).

\section{Lemmas}

For the proof of these theorems, we need the following lemmas.

Lemma 2.1. If $P \in P_{n, \mu}$ and $Q(z)=z^{n} \overline{P\left(\frac{1}{\bar{z}}\right)}$, then

$$
\left|Q^{\prime}(z)\right| \leq s_{\mu}\left|P^{\prime}(z)\right| \text { for }|z|=1,
$$

where $s_{\mu}$ is defined by (1.9).

The above lemma is due to Aziz and Rather [3]. 
Lemma 2.2. If $P \in P_{n, 1}$ with $k>0$ and $Q(z)=z^{n} \overline{P\left(\frac{1}{\bar{z}}\right)}$, then $|Q(z)| \geq \frac{m}{k^{n}}$ for $|z| \leq \frac{1}{k}$ and in particular

$$
\left|a_{n}\right|>\frac{m}{k^{n}} \text {. }
$$

Lemma 2.3. If $P \in P_{n, \mu}$, then

$$
A_{\mu} \leq k^{\mu}
$$

where $A_{\mu}$ is defined by the formula (1.12).

Lemma 2.4. If $P \in P_{n, \mu}$ and $Q(z)=z^{n} \overline{P\left(\frac{1}{\bar{z}}\right)}$, then on $|z|=1$

$$
\left|Q^{\prime}(z)\right| \leq k^{\mu}\left|P^{\prime}(z)\right|-\frac{n m}{k^{n-\mu}} .
$$

The above Lemmas 2.2, 2.3 and 2.4 are all due to Dewan et.al. [9].

Lemma 2.5. If $P \in P_{n, \mu}$, then for every complex number $\alpha$ with $|\alpha| \geq k^{\mu}$,

$$
\left|D_{\alpha} P(z)\right| \geq \frac{|\alpha| m n}{k^{n}} \text { for }|z|=1 \text {. }
$$

Proof of Lemma 2.5. If $P$ has a zero on $|z|=k$ then $m=0$ and in this case Lemma 2.5 holds trivially. Hence we suppose that all the zeros of $P$ lie in $|z|<k, k \leq 1$ so that $m>0$. Now $m \leq|P(z)|$ for $|z|=k$, therefore it follows by Rouche's theorem that for every complex number $\lambda$ with $|\lambda|<1$, the polynomial $P-\frac{m \lambda z^{n}}{k^{n}}$ has all its zeros in $|z|<k, k \leq 1$, therefore,by Guass-Lucas theorem, the polynomial $P^{\prime}-\frac{m n \lambda z^{n-1}}{k^{n}}$ also has all its zeros in $|z|<k k \leq 1$ and hence

$$
\left|P^{\prime}(z)\right| \geq \frac{m n|z|^{n-1}}{k^{n}} \text { for }|z| \geq k .
$$

Because, if (2.5) is not true, then there is a point $z=z_{0}$ with $\left|z_{0}\right| \geq k$ such that

$$
\left|P^{\prime}\left(z_{0}\right)\right|<\frac{m n\left|z_{0}\right|^{n-1}}{k^{n}} .
$$

If we take $\lambda=\frac{k^{n} P^{\prime}\left(z_{0}\right)}{m n z_{0}^{n-1}}$, so that $|\lambda|<1$, then we have

$$
P^{\prime}\left(z_{0}\right)-\frac{m n \lambda z_{0}^{n-1}}{k^{n}}=0
$$

where $\left|z_{0}\right| \geq k$, which contradicts to the fact that all the zeros of $P^{\prime}-\frac{m n \lambda z^{n-1}}{k^{n}}$ lie in $|z|<k, k \leq 1$.

Also for $|z|=1$, we have

$$
\begin{aligned}
\left|D_{\alpha} P(z)\right| & =\left|n P(z)+(\alpha-z) P^{\prime}(z)\right| \\
& \geq|\alpha|\left|P^{\prime}(z)\right|-\left|n P(z)-z P^{\prime}(z)\right| \\
& =|\alpha|\left|P^{\prime}(z)\right|-\left|Q^{\prime}(z)\right| .
\end{aligned}
$$

Combining this with inequality (2.4) of Lemma 2.4, we get for $|z|=1$ and $|\alpha| \geq k^{\mu}$,

$$
\left|D_{\alpha} P(z)\right| \geq\left(|\alpha|-k^{\mu}\right)\left|P^{\prime}(z)\right|+\frac{m n}{k^{n-\mu}} .
$$

Inequality (2.7) in conjunction with (2.5) gives for $|z|=1$ and $|\alpha| \geq k^{\mu}$,

$$
\left|D_{\alpha} P(z)\right| \geq \frac{|\alpha| m n}{k^{n}} .
$$

This completes the proof of Lemma 2.5. 
Lemma 2.6. If $P \in P_{n, \mu}$, then

$$
\frac{m}{k^{n}} \leq\|P\|_{\infty} .
$$

Proof of Lemma 2.6. Since $P$ has all its zeros in $|z| \leq k, k \leq 1$, we have from Lemma 2.4,

$$
\left|Q^{\prime}(z)\right| \leq k^{\mu}\left|P^{\prime}(z)\right|-\frac{m n}{k^{n-\mu}} \text { for }|z|=1 .
$$

On using (1.1) in above inequality, we get for $|z|=1$,

$$
\begin{aligned}
\left|Q^{\prime}(z)\right| & \leq k^{\mu} n\|P\|_{\infty}-\frac{m n}{k^{n-\mu}} \\
& =n k^{\mu}\left\{\|P\|_{\infty}-\frac{m}{k^{n}}\right\},
\end{aligned}
$$

which is true and this proves Lemma 2.6.

Lemma 2.7. The function

$$
s_{\mu}(x)=\frac{n x k^{2 \mu}+\mu\left|a_{n-\mu}\right| k^{\mu-1}}{n x k^{\mu-1}+\mu\left|a_{n-\mu}\right|}
$$

where $k \leq 1, \mu \geq 1$, is a non-increasing function of $x$.

Proof of Lemma 2.7. The proof is simple and follows by first derivative test.

\section{Proofs of the theorems}

Proof of Theorem 1.1. Let $Q=z^{n} \overline{P\left(\frac{1}{\bar{z}}\right)}$, then $P=z^{n} \overline{Q\left(\frac{1}{\bar{z}}\right)}$ and it can be easily verified that for $|z|=1$,

$$
\left|Q^{\prime}(z)\right|=\left|n P(z)-z P^{\prime}(z)\right|
$$

and

$$
\left|P^{\prime}(z)\right|=\left|n Q(z)-z Q^{\prime}(z)\right| .
$$

Since $P$ has all its zeros in $|z| \leq k$, therefore, by using Lemma 2.1 and (3.2), we have for $|z|=1$,

$$
\left|Q^{\prime}(z)\right| \leq s_{\mu}\left|n Q(z)-z Q^{\prime}(z)\right| .
$$

Now for every $\alpha \in \mathbb{C}$ with $|\alpha| \geq s_{\mu}$, we have

$$
\begin{aligned}
\left|D_{\alpha} P(z)\right| & =\left|n P(z)+(\alpha-z) P^{\prime}(z)\right| \\
& \geq|\alpha|\left|P^{\prime}(z)\right|-\left|n P(z)-z P^{\prime}(z)\right|,
\end{aligned}
$$

which on using (3.1) and Lemma 2.1 gives for $|z|=1$,

$$
\begin{aligned}
\left|D_{\alpha} P(z)\right| & \geq|\alpha|\left|P^{\prime}(z)\right|-\left|Q^{\prime}(z)\right| \\
& \geq\left(|\alpha|-s_{\mu}\right)\left|P^{\prime}(z)\right| .
\end{aligned}
$$

Again since $P$ has all its zeros in $|z| \leq k, k \leq 1$, it follows by Guass-Lucas theorem that all the zeros of $P^{\prime}$ also lie in $|z| \leq k, k \leq 1$. This implies that the polynomial

$$
z^{n-1} \overline{P\left(\frac{1}{\bar{z}}\right)}=n Q-z Q^{\prime}
$$

has all its zeros in $|z| \geq \frac{1}{k} \geq 1$. Therefore, it follows from (3.3) that the function

$$
W(z)=\frac{z Q^{\prime}(z)}{s_{\mu}\left(n Q(z)-z Q^{\prime}(z)\right)}
$$


is analytic for $|z| \leq 1$ and $|W(z)| \leq 1$ for $|z| \leq 1$. Furthermore, $W(0)=0$ and so the function $1+s_{\mu} W(z)$ is subordinate to the function $1+s_{\mu} z$ for $|z| \leq 1$. Hence by a well-known property of sub-ordination [14], we have for each $\gamma>0$,

$$
\int_{0}^{2 \pi}\left|1+s_{\mu} W\left(e^{i \theta}\right)\right|^{\gamma} d \theta \leq \int_{0}^{2 \pi}\left|1+s_{\mu} e^{i \theta}\right|^{\gamma} d \theta
$$

Now

$$
1+s_{\mu} W(z)=\frac{n Q(z)}{n Q(z)-z Q^{\prime}(z)},
$$

which gives with the help of (3.2) that for $|z|=1$

$$
n|Q(z)|=\left|1+s_{\mu} W(z)\right|\left|P^{\prime}(z)\right| .
$$

Since $|P(z)|=|Q(z)|$ for $|z|=1$, therefore from (3.6), we get

$$
\left|P^{\prime}(z)\right|=\frac{n|P(z)|}{\left|1+s_{\mu} W(z)\right|} \text { for }|z|=1
$$

From (3.4) and (3.7), we deduce that for each $\gamma>0$,

$$
n^{\gamma}\left(|\alpha|-s_{\mu}\right)^{\gamma} \int_{0}^{2 \pi}\left|P\left(e^{i \theta}\right)\right|^{\gamma} d \theta \leq \int_{0}^{2 \pi}\left|1+s_{\mu} W\left(e^{i \theta}\right)\right|^{\gamma}\left|D_{\alpha} P\left(e^{i \theta}\right)\right|^{\gamma} d \theta .
$$

This gives with the help of Holder's inequality for $p>1, q>1$ with $p^{-1}+q^{-1}=1$, that

$$
\begin{aligned}
n^{\gamma}\left(|\alpha|-s_{\mu}\right)^{\gamma} & \int_{0}^{2 \pi}\left|P\left(e^{i \theta}\right)\right|^{\gamma} d \theta \\
& \leq\left\{\int_{0}^{2 \pi}\left|1+s_{\mu} W\left(e^{i \theta}\right)\right|^{p \gamma} d \theta\right\}^{\frac{1}{p}}\left\{\int_{0}^{2 \pi}\left|D_{\alpha} P\left(e^{i \theta}\right)\right|^{q \gamma} d \theta\right\}^{\frac{1}{q}} .
\end{aligned}
$$

The above inequality in conjunction with (3.5) gives

$$
\begin{aligned}
n\left(|\alpha|-s_{\mu}\right) & \left\{\int_{0}^{2 \pi}\left|P\left(e^{i \theta}\right)\right|^{\gamma} d \theta\right\}^{\frac{1}{\gamma}} \\
& \leq\left\{\int_{0}^{2 \pi}\left|1+s_{\mu} e^{i \theta}\right|^{p \gamma} d \theta\right\}^{\frac{1}{p \gamma}}\left\{\int_{0}^{2 \pi}\left|D_{\alpha} P\left(e^{i \theta}\right)\right|^{q \gamma} d \theta\right\}^{\frac{1}{q \gamma}},
\end{aligned}
$$

which completes the proof of Theorem 1.1.

Proof of Theorem 1.2. If $P$ has a zero on $|z|=k$, then $m=0$ and the result follows from Theorem 1.1 in this case. Hence forth, we suppose that all the zeros of $P$ lie in $|z|<k, k \leq 1$, so that $m>0$. Now $m \leq|P(z)|$ for $|z|=k$, therefore if $\beta$ is any complex number with $|\beta|<1$, then

$$
\left|\frac{m \beta z^{n}}{k^{n}}\right|<|P(z)| \text { for }|z|=k .
$$

Since all the zeros of $P$ lie in $|z|<k$, it follows by Rouche's theorem that all the zeros of $F=P-\frac{m \beta z^{n}}{k^{n}}$ also lie in $|z|<k$. If $G=z^{n} \overline{F\left(\frac{1}{\bar{z}}\right)}=Q-\frac{m \bar{\beta}}{k^{n}}$, then it can be easily verified that for $|z|=1$,

$$
\left|F^{\prime}(z)\right|=\left|n G(z)-z G^{\prime}(z)\right|
$$


and

$$
\left|G^{\prime}(z)\right|=\left|n F(z)-z F^{\prime}(z)\right| .
$$

As $F$ has all its zeros in $|z|<k, k \leq 1$, the inequality (2.1) of Lemma 2.1 in conjunction with (3.8) gives

$$
\left|G^{\prime}(z)\right| \leq s_{\mu}^{\prime}\left|n G(z)-z G^{\prime}(z)\right| \text { for }|z|=1
$$

where

$$
s_{\mu}^{\prime}=\frac{n\left|a_{n}-\frac{m \beta}{k^{n}}\right| k^{2 \mu}+\mu\left|a_{n-\mu}\right| k^{\mu-1}}{n\left|a_{n}-\frac{m \beta}{k^{n}}\right| k^{\mu-1}+\mu\left|a_{n-\mu}\right|} .
$$

Since for every $\beta$ with $|\beta|<1$, we have

$$
\left|a_{n}-\frac{m \beta}{k^{n}}\right| \geq\left|a_{n}\right|-\frac{m|\beta|}{k^{n}} \geq\left|a_{n}\right|-\frac{m}{k^{n}}
$$

and $\left|a_{n}\right|>\frac{m}{k^{n}}$ by Lemma 2.2, it follows by combining (3.11), (3.12) and Lemma 2.7 for every $\beta$ with $|\beta|<1$, that

$$
\begin{aligned}
s_{\mu}^{\prime} & =\frac{n\left|a_{n}-\frac{m \beta}{k^{n}}\right| k^{2 \mu}+\mu\left|a_{n-\mu}\right| k^{\mu-1}}{n\left|a_{n}-\frac{m \beta}{k^{n}}\right| k^{\mu-1}+\mu\left|a_{n-\mu}\right|} \\
& \leq \frac{n\left(\left|a_{n}\right|-\frac{m}{k^{n}}\right)\left|k^{2 \mu}+\mu\right| a_{n-\mu} \mid k^{\mu-1}}{n\left(\left|a_{n}\right|-\frac{m}{k^{n}}\right) k^{\mu-1}+\mu\left|a_{n-\mu}\right|}=A_{\mu} .
\end{aligned}
$$

Using this in (3.10), we get

$$
\left|G^{\prime}(z)\right| \leq A_{\mu}\left|n G(z)-z G^{\prime}(z)\right| \text { for }|z|=1 .
$$

Now for every complex number $\alpha$ with $|\alpha| \geq A_{\mu}$, we have

$$
\begin{aligned}
\left|D_{\alpha} F(z)\right| & =\left|n F(z)+(\alpha-z) F^{\prime}(z)\right| \\
& \geq|\alpha|\left|F^{\prime}(z)\right|-\left|n F(z)-z F^{\prime}(z)\right| .
\end{aligned}
$$

On combining inequalities (3.8), (3.9), (3.14) and (3.15), we get for $|z|=1$ and $|\alpha| \geq A_{\mu}$,

$$
\left|D_{\alpha} F(z)\right| \geq\left(|\alpha|-A_{\mu}\right)\left|F^{\prime}(z)\right|
$$

which implies,

$$
\left|D_{\alpha} P(z)-\frac{n m \alpha \beta z^{n-1}}{k^{n}}\right| \geq\left(|\alpha|-A_{\mu}\right)\left|P^{\prime}(z)-\frac{n m \beta z^{n-1}}{k^{n}}\right| \text { for }|z|=1 .
$$

Again since $F$ has all its zeros in $|z|<k, k \leq 1$, it follows by Guass-Lucas theorem that all the zeros of the polynomial $F^{\prime}$ also lie in $|z|<k, k \leq 1$. Therefore the polynomial $z^{n-1} \overline{F^{\prime}\left(\frac{1}{\bar{z}}\right)}=n G-z G^{\prime}$ has all its zeros in $|z|>\frac{1}{k} \geq 1$. Hence it follows from (3.14) that the function

$$
W(z)=\frac{z G^{\prime}(z)}{A_{\mu}\left(n G(z)-z G^{\prime}(z)\right)}
$$

is analytic for $|z| \leq 1$ with $|W(z)| \leq 1$ for $|z| \leq 1$ and $W(0)=0$. Thus the function $1+A_{\mu} W(z)$ is subordinate to the function $1+A_{\mu} z$ for $|z| \leq 1$. By a well known property of sub-ordination (see [14]), we have for each $\gamma>0$,

$$
\int_{0}^{2 \pi}\left|1+A_{\mu} W\left(e^{i \theta}\right)\right|^{\gamma} d \theta \leq \int_{0}^{2 \pi}\left|1+A_{\mu} e^{i \theta}\right|^{\gamma} d \theta .
$$


Now by (3.18), we have

$$
1+A_{\mu} W(z)=\frac{n G(z)}{n G(z)-z G^{\prime}(z)},
$$

which gives with the help of (3.8) that for $|z|=1$,

$$
n|G(z)|=\left|1+A_{\mu} W(z)\right|\left|F^{\prime}(z)\right| .
$$

Since $|G(z)|=|F(z)|$ for $|z|=1$, therefore above equation reduces to

$$
\left|F^{\prime}(z)\right|=\frac{n|F(z)|}{\left|1+A_{\mu} W(z)\right|} \text { for }|z|=1,
$$

or

$$
\frac{n\left|P(z)-\frac{m \beta z^{n}}{k^{n}}\right|}{\left|1+A_{\mu} W(z)\right|}=\left|P^{\prime}(z)-\frac{n m \beta z^{n-1}}{k^{n}}\right| \text { for }|z|=1 .
$$

The above equation (3.20) with the help of (3.17) gives for $|z|=1$ and $|\alpha| \geq A_{\mu}$,

$$
n\left(|\alpha|-A_{\mu}\right)\left|P(z)-\frac{m \beta z^{n}}{k^{n}}\right| \leq\left|1+A_{\mu} W(z)\right|\left|D_{\alpha} P(z)-\frac{\alpha n m \beta z^{n-1}}{k^{n}}\right| .
$$

From (3.21), we have for each $\gamma>0$,

$$
\begin{aligned}
n^{\gamma}\left(|\alpha|-A_{\mu}\right)^{\gamma} & \int_{0}^{2 \pi}\left|P\left(e^{i \theta}\right)-\frac{m \beta e^{i n \theta}}{k^{n}}\right|^{\gamma} d \theta \\
& \leq \int_{0}^{2 \pi}\left|1+A_{\mu} W\left(e^{i \theta}\right)\right|^{\gamma}\left|D_{\alpha} P\left(e^{i \theta}\right)-\frac{n m \alpha \beta e^{i(n-1) \theta}}{k^{n}}\right|^{\gamma} d \theta .
\end{aligned}
$$

This gives with the help of Holder's inequality for $p>1, q>1$ with $p^{-1}+q^{-1}=1$,

$$
\begin{aligned}
n^{\gamma}\left(|\alpha|-A_{\mu}\right)^{\gamma} & \int_{0}^{2 \pi}\left|P\left(e^{i \theta}\right)-\frac{m \beta e^{i n \theta}}{k^{n}}\right|^{\gamma} d \theta \\
& \leq\left\{\int_{0}^{2 \pi}\left|1+A_{\mu} W\left(e^{i \theta}\right)\right|^{p \gamma}\right\}^{\frac{1}{p}}\left\{\int_{0}^{2 \pi}\left|D_{\alpha} P\left(e^{i \theta}\right)-\frac{n m \alpha \beta e^{i(n-1) \theta}}{k^{n}}\right|^{q \gamma} d \theta\right\}^{\frac{1}{q}} .
\end{aligned}
$$

Now using (3.19) with $\gamma$ replaced by $p \gamma$ in (3.22), we obtain for each $\gamma>0, p>1, q>1$ with $p^{-1}+q^{-1}=1$,

$$
\begin{aligned}
n^{\gamma}\left(|\alpha|-A_{\mu}\right)^{\gamma} & \int_{0}^{2 \pi}\left|P\left(e^{i \theta}\right)-\frac{m \beta e^{i n \theta}}{k^{n}}\right|^{\gamma} d \theta \\
& \leq\left\{\int_{0}^{2 \pi}\left|1+A_{\mu} e^{i \theta}\right|^{p \gamma}\right\}^{\frac{1}{p}}\left\{\int_{0}^{2 \pi}\left|D_{\alpha} P\left(e^{i \theta}\right)-\frac{n m \alpha \beta e^{i(n-1) \theta}}{k^{n}}\right|^{q \gamma} d \theta\right\}^{\frac{1}{q}} .
\end{aligned}
$$

Equivalently,

$$
\begin{aligned}
n\left(|\alpha|-A_{\mu}\right) & \left\{\int_{0}^{2 \pi}\left|P\left(e^{i \theta}\right)-\frac{m \beta e^{i n \theta}}{k^{n}}\right|^{\gamma} d \theta\right\}^{\frac{1}{\gamma}} \\
& \leq\left\{\int_{0}^{2 \pi}\left|1+A_{\mu} e^{i \theta}\right|^{p \gamma}\right\}^{\frac{1}{p \gamma}}\left\{\int_{0}^{2 \pi}\left|D_{\alpha} P\left(e^{i \theta}\right)-\frac{n m \alpha \beta e^{i(n-1) \theta}}{k^{n}}\right|^{q \gamma} d \theta\right\}^{\frac{1}{q \gamma}},
\end{aligned}
$$

which is equivalent to inequality (1.16) and this completes the proof of Theorem 1.2. 


\section{Further Remarks}

Remark 3.1. If we make use of inequality (2.3) and the fact that

$\left\|1+A_{\mu} z\right\|_{\gamma} \leq\left\|1+k^{\mu} z\right\|_{\gamma}, \gamma>0$

we immediately get the following useful consequences from Theorem 1.2.

Corollary 3.1. If $P \in P_{n, \mu}$, then for every complex numbers $\alpha, \beta$ with $|\alpha| \geq k^{\mu},|\beta|<1$ and for each $\gamma>0, p>1, q>1$ with $p^{-1}+q^{-1}=1$, we have

$$
n\left(|\alpha|-A_{\mu}\right)\left\|P-\frac{m \beta z^{n}}{k^{n}}\right\|_{\gamma} \leq\left\|1+k^{\mu} z\right\|_{p \gamma}\left\|D_{\alpha} P-\frac{\alpha \beta m n z^{n-1}}{k^{n}}\right\|_{q \gamma},
$$

where $A_{\mu}$ is defined by (1.12).

Corollary 3.2. If $P \in P_{n, \mu}$, then for every complex numbers $\alpha, \beta$ with $|\alpha| \geq k^{\mu},|\beta|<1$ and for each $\gamma>0, p>1, q>1$ with $p^{-1}+q^{-1}=1$, we have

$$
n\left(|\alpha|-k^{\mu}\right)\left\|P-\frac{m \beta z^{n}}{k^{n}}\right\|_{\gamma} \leq\left\|1+k^{\mu} z\right\|_{p \gamma}\left\|D_{\alpha} P-\frac{\alpha \beta m n z^{n-1}}{k^{n}}\right\|_{q \gamma} .
$$

Remark 3.2. Making $\gamma \rightarrow \infty$ and $p \rightarrow \infty$ (so that $q \rightarrow 1$ ) in (3.23) and (3.24) and using the same arguments as in Remark 1.2, we get inequalities (1.11) and (1.10), respectively.

\section{Acknowledgment}

The work is sponsored by UGC, Govt. of India under the Major Research Project Scheme vide no. MRP-MAJORMATH-2013-29143.

\section{References}

[1] A. Mir and B. Dar., Some inequalities concerning the polar derivative of a polynomial-II, Anal. Theory Appl., 29 (2013), 384-389.

[2] A. Aziz and Q. M. Dawood., Inequalities for a polynomial and its derivative, J. Approx. Theory, 54 (1988), 306-313.

[3] A. Aziz and N. A. Rather., Some Zygmund type $L^{q}$-inequalities for polynomials, J. Math. Anal. Appl., 289 (2004), 14-29.

[4] A.Aziz and N.A.Rather., Inequalities for the polar derivative of a polynomial with restricted zeros, Math. Balk., 17 (2003), 15-28.

[5] A. Aziz and N. A. Rather., A refinement of a theorem of Paul Turan concerning polynomials, Math. Ineq. Appl., 1(1998), 231-238.

[6] A. Aziz and W. M. Shah., An integral mean estimate for polynomials, Indian J. Pure Appl. Math., 28 (1997), 1413-1419.

[7] S. Bernstein., Lecons Sur Les Proprietes extremals et la meilleure approximation des fonctions analytiques d'une fonctions reelle, Gauthier-villars (Paris 1926).

[8] K. K. Dewan, N. Singh and R. Lal., Inequalities for the polar derivative of a polynomial, Int. J. Pure. Appl. Math., 33 (2006), 109-117.

[9] K. K. Dewan, N. Singh and A. Mir., Extensions of some polynomial inequalities to the polar derivative, J. Math. Anal. Appl., 352 (2009), 807-815.

[10] K. K. Dewan, A. Mir and R. S. Yadav., Integral mean estimates for polynomials whose zeros are with in a circle, IJMMS, 4 (2001), 231-235.

[11] K. K. Dewan, N. Singh, A. Mir and A. Bhat., Some inequalities for the polar derivative of a polynomial, Southeast Asain Bull. Math., 34 (2010), 69-77. 
[12] N. K. Govil., Some inequalities for derivative of polynomials, J.Approx. Theory, 66 (1991), 29-35.

[13] N. K. Govil, Q. I. Rahman and G. Schemeisser., On the derivative of a polynomial, Illinois J. Math., 23 (1979), 319-330.

[14] E. Hille, Ananlytic function theory, Vol II, Ginn and Company, New York, Toranto, 1962.

[15] M. A. Malik., On the derivative of a polynomial, J. London Math. Soc., 1 (1969), 57-60.

[16] M. Riesz., Eine trigonometrische interpolationsformel und einige Ungleichungen für Polynome, Jahresbericht der Deutschen Mathematiker-Vereinigung, 23 (1914), 354-368.

[17] P. Turán., Über die ableitung von polynomen, Compositio Math., 7 (1939), 89-95.

\section{Affiliations}

AbDullah Mir

ADDRESS: University of Kashmir, Dept. of Mathematics, 190006, Srinagar-India.

E-MAIL: abdullahmir122196@gmail.com 